\title{
Turbulent fluctuations above the buffer layer of wall-bounded flows
}

\author{
JAVIER JIMÉNEZ ${ }^{1,2}$ AND SERGIO HOYAS ${ }^{1} \uparrow$ \\ ${ }^{1}$ School of Aeronautics, Universidad Politécnica de Madrid, 28040 Madrid, Spain \\ ${ }^{2}$ Centre for Turbulence Research, Stanford University, Stanford, CA 94305, USA
}

(Received 15 July 2007 and in revised form 21 May 2008)

The behaviour of the velocity and pressure fluctuations in the logarithmic and outer layers of turbulent flows is analysed using spectral information and probability density functions from channel simulations at $R e_{\tau} \leqslant 2000$. Comparisons are made with experimental data at higher Reynolds numbers. It is found, in agreement with previous investigations, that the intensity profiles of the streamwise and spanwise velocity components have logarithmic ranges that are traced to the widening spectral range of scales as the wall is approached. The same is true for the pressure, both theoretically and observationally, but not for the normal velocity or for the tangential stress cospectrum, although even those two quantities have structures with lengths of the order of several hundred times the wall distance. Because the logarithmic range grows longer as the Reynolds number increases, variables which are 'attached' in this sense scale in the buffer layer in mixed units. These results give strong support to the attached-eddy scenario proposed by Townsend (1976), but they are not linked to any particular eddy model. The scaling of the outer modes is also examined. The intensity of the streamwise velocity at fixed $y / h$ increases with the Reynolds number. This is traced to the large-scale modes, and to an increased intensity of the ejections but not of the sweeps. Several differences are found between the outer structures of different flows. The outer modes of the spanwise and wall-normal velocities in boundary layers are stronger than in internal flows, and their streamwise velocities penetrate closer to the wall. As a consequence, their logarithmic layers are thinner, and some of their logarithmic slopes are different. The channel statistics are available electronically at http://torroja.dmt.upm.es/ftp/channels/.

\section{Introduction}

Although the behaviour of turbulence in the buffer layer of wall-bounded flows has been exhaustively studied in the last decade, the logarithmic and outer layers have been addressed less often. They were nevertheless the parts of the flow for which a theory was first proposed. The best-known prediction, and the one that has been discussed most, is the logarithmic profile of the mean velocity in the overlap layer between the inner and outer flow regions. We will not address that question here at all. A summary of its early history is given in the book by Schlichting (1968), and a flavour of the current controversies surrounding it can be found in Zagarola, Perry \&

\footnotetext{
$\dagger$ Present address: Department of Informatics, Univ. de Valencia, 46100 Burjassot, Valencia, Spain.
} 
Smits (1997), Barenblatt, Chorin \& Prostokishin (2000), or Wosnik, Castillo \& George (2000).

The first goal of this paper is to examine the evidence for logarithmic profiles of the fluctuating turbulent quantities in the overlap layer. Such profiles were first predicted by Townsend $(1976, \S 5.7-5.8)$, and the argument has been especially developed by the Melbourne group within a particular model of 'hairpin' eddies (Perry, Henbest \& Chong 1986; Perry \& Li 1990). One of the reasons why they are interesting is because any quantity with such a profile cannot satisfy the other well-known prediction of the classical theory, which is that the friction velocity $u_{\tau}$ is a uniform velocity scale across the flow. If we define wall units in terms of $u_{\tau}$ and of the fluid viscosity $v$, and denote by a + superscript any variable expressed in them, the overlap layer extends from a lower limit that scales in wall units, to an outer one that scales with the flow thickness $h$. Any quantity that behaves like $\log y$, where $y$ is the distance to the wall, increases across that range by an amount proportional to $\log h^{+} \equiv \log R e_{\tau}$. Therefore, there can not be a scale that is independent of the Reynolds number and that applies at the same time to its inner and to its outer values.

While Townsend's argument has most often been discussed in terms of the hairpin model, it was originally independent of those details. In this paper we examine the information that can be obtained from the kinematics of the flow without any particular dynamical model in mind. For example, we will be interested in whether the predicted logarithmic profiles are universal across different flows and Reynolds numbers. The dynamics themselves require further work, and are left for future publications.

A second goal of the paper is to inquire about the universality of the scaling of the fluctuations above the logarithmic layer. Townsend's argument says nothing against the classical $u_{\tau}$ scaling at wall distances that are fixed fractions of the flow thickness, but there is increasing evidence that that part of the flow is not universal either. That evidence will be discussed and, as far as possible, interpreted.

We use mostly data from recent simulations of turbulent channels, with emphasis on their spectra and on quantities, such as the pressure, which are difficult to obtain otherwise. To alleviate the Reynolds-number limitation of the simulations, we put them whenever possible in the context of higher-Reynolds-number experiments.

The paper is organized as follows. The numerical simulations are described in $\S 2$. Our interpretation of the classical spectral theory of the logarithmic layer, and the nomenclature used in the paper, are presented in $\S 3$. The velocity fluctuations are discussed in $\S 4$. The pressure fluctuations are analysed in $\S 5$, and $\S 6$ concludes.

\section{The numerical and experimental data}

Most of the detailed discussion in this paper is based on the turbulent channel simulations of del Álamo \& Jiménez (2003), del Álamo et al. (2004) and Hoyas \& Jiménez (2006). Their parameters are summarized in table 1 . The numerical codes integrate the Navier-Stokes equations in the form of evolution problems for the wallnormal vorticity $\omega_{y}$ and for the Laplacian of the wall-normal velocity $\nabla^{2} v$, as in Kim, Moin \& Moser (1987). The spatial discretization uses de-aliased Fourier expansions in the wall-parallel planes. The two lowest Reynolds numbers use Chebyschev polynomials in the wall-normal coordinate, and the highest one uses seven-point compact finite differences. The characteristics of the different simulations with respect to the large scales were documented in the original publications. Their resolution properties regarding the smallest scales were discussed by del Álamo et al. (2006). 


\begin{tabular}{lccccccccc}
\hline Case & Line & $R e_{\tau}$ & $L_{x} / h$ & $L_{z} / h$ & $\Delta x^{+}$ & $\Delta z^{+}$ & $N_{y}$ & $N_{F}$ & $u_{\tau} T / h$ \\
L550 & --- & 547 & $8 \pi$ & $4 \pi$ & 13 & 6.7 & 257 & 149 & 22 \\
L950 & --- & 934 & $8 \pi$ & $3 \pi$ & 11 & 5.7 & 385 & 74 & 12 \\
L2000 & - & 2003 & $8 \pi$ & $3 \pi$ & 12 & 6.1 & 633 & 226 & 11
\end{tabular}

TABLE 1. Parameters of the simulations. $L_{x}$ and $L_{z}$ are the periodic streamwise and spanwise dimensions of the numerical box, and $h$ is the channel half-width. $\Delta x$ and $\Delta z$ are the resolutions in terms of Fourier modes. $N_{y}$ is the number of wall-normal collocation points. $N_{F}$ is the number of fields used to accumulate statistics, and $T$ is the time spanned by those fields.

Because of minor bugs discovered in L550 and L950 after their original publication, their statistics have been recomputed by rerunning each of the stored fields for a few hundred time steps with a clean code. The occasion was used to extend the run of L550, for which relatively few fields had been stored, by several extra eddy turnovers, as well as to compute the budgets of the Reynolds-stress tensor for the three cases (Hoyas \& Jiménez 2008). The difference between the new and the older statistics was found to be negligible except below $y^{+} \approx 1$, which was the location of the most important bug. The statistics of L2000 were, as far as we know, bug-free.

We use $x, z$ and $u, w$, respectively, for the streamwise and spanwise coordinates and velocity components. The kinematic pressure $p$ incorporates the constant fluid density, which is also left out of the definition of the Reynolds stresses. The flow thickness $h$ is the half-width for channels, the radius for pipes, and the $99 \%$ thickness for boundary layers. Primed quantities refer to root-mean-squared intensities of the fluctuations. Otherwise, capital letters refer to mean quantities, and lower-case ones to fluctuations. The brackets \langle\rangle represent averaging over homogeneous directions, usually over wall-parallel planes and time.

In presenting spectral data, the wavenumbers in the two wall-parallel directions are $k_{x}$ and $k_{z}$, and the corresponding wavelengths are $\lambda=2 \pi / k$. The premultiplied spectral energy densities, $\phi$, are defined in terms of the one- or two-dimensional spectra, $E$, as $\phi_{*}=k_{x} E_{*}\left(k_{x}\right)$ or $k_{x} k_{z} E_{*}\left(k_{x}, k_{z}\right)$, where the asterisk stands for any two flow fields, such as $u v$ or $p p$. Whether one- or two-dimensional quantities are implied will always be clear from the context.

Even if, to our knowledge, the Reynolds numbers of our simulations are among the highest available, they are relatively low to allow any decoupling that may exist between the outer and the inner layers, or to ensure the existence of a logarithmic layer. On the other hand, their advantage is that they span a relatively wide range of Reynolds numbers using uniform numerical methods in comparable grids and computational domains. They are therefore well adapted to study trends.

We have supplemented them whenever possible with higher-Reynolds-number experiments, but, since high-Reynolds-number experimental channels are rare, and since it is unclear whether the outer layers of different flows can be assumed to be similar, we always use in the figures different symbols for boundary layers $(\nabla)$, pipes $(\triangle)$, and channels $(O)$. All the data used here are available from the respective authors, although some of them may be difficult to find in electronic form. The note at the end of some references refers to their case numbers in the electronic data compilation by AGARD (1998). The data originating from our group are available at http://torroja.dmt.upm.es/ftp/channels/.

A word should be said about statistical uncertainty. Error estimates were not available for most experimental data, but our channels are compiled over several 
hundred short numerical runs, and the final statistical errors can be estimated from the scatter among the individual statistics, and from how they evolve in time. The details can be found in the Appendix to Hoyas \& Jiménez (2008), and the standard deviations for all the one-point statistics are in the web page above. They are typically well below $1 \%$. The uncertainties of other quantities are harder to define. For example, in the spectra discussed in figure 2 , or in the probability density functions (p.d.f.s) in figure 7, both of which are essentially histograms, the uncertainty depends on the size of the averaging bins being considered. The spectra, for example, have their largest uncertainties for the longest and widest scales, which represent large structures with long lifetimes. Applying the procedure above, it is found that the worse relative scatter over spectral boxes defined by wavelengths spaced by a factor of two has a standard deviation of the order of $\pm 10 \%$. It is generally below $\pm 5 \%$, and becomes much smaller when either of the wavelengths is small with respect to the channel width. The p.d.f.s in figure 7 are compiled using approximately $10^{9}$ points at each wall distance, so that the outermost $10^{-4}$ isolines in figure $7(b)$ represent about $10^{5}$ points. In all cases our data are plotted without smoothing, so that the uncertainties can be roughly estimated from the smoothness of the lines.

\section{The classical theory}

In this section we review the classical arguments on the behaviour of the turbulent fluctuations in the overlap layer, partly as a reference, but also to define the nomenclature used in the rest of the paper. Much of the section is adapted from chapter 5 of the book by Townsend (1976).

The minimal condition for the existence of an overlap layer is that there exists a region too far from the wall for viscosity to be important, but close enough to it for the total tangential stress not to be very different from its value $u_{\tau}^{2}$ at the wall. The viscous stresses are negligible in such a layer, and the averaged tangential Reynolds stress is $-\langle u v\rangle$. The only 'possible' velocity scale is then $u_{\tau}$.

From the point of view of this paper, an eddy is defined as a logarithmically spaced band of wall-parallel wavelengths, such as between $\lambda_{0} / 2$ and $\lambda_{0}$, with its associated wall-normal distribution. The latter provides a wall-normal location and dimension for the eddy, such as the centre of gravity and the radius of inertia of the wall-normal distribution of the velocity magnitude. In principle, an eddy refers to a single flow variable, but it is often possible to identify overlapping structures of the three velocity components as forming part of the 'same' eddy.

What Townsend observed was that turbulent eddies centred at a distance $y$ from the wall should behave differently depending on whether their sizes are larger or smaller than $y$. 'Detached' eddies whose wall-normal dimensions are smaller than $y$ feel the presence of the wall only indirectly (e.g. through the local shear of the mean profile) and therefore behave more or less as in free shear flows. They tend to be roughly isotropic and to form classical Kolmogorov (1941) energy cascades. On the general argument that the energy spectrum of a turbulent flow decreases with increasing wavenumber, the strongest detached eddies should be those with sizes roughly equal to $y$. For variables such as the wall-normal velocity, those 'maximal' detached eddies are also the largest ones, because of the impermeability condition. Wall-parallel velocities, on the other hand, are not constrained in this way, and can have much larger lateral dimensions. Those 'attached' eddies are not isotropic, and, since they do not contain $v$, they cannot be 'active' near the wall in the sense of carrying tangential Reynolds stresses. 
The argument for logarithmic profiles of the intensities was also made by Townsend (1976, pp. 150-154) in his original formulation of the attached-eddy model, and has been incorporated in most later models (Perry et al. 1986; Perry \& Li 1990; Kunkel \& Marusic 2006). It is weaker than that for a uniform velocity scale. The idea is that all eddies are active somewhere, so that the only attached eddies are the maximal detached ones. At a given wall distance, eddies with wall-parallel sizes $\lambda \gg y$ are wall-parallel sections of larger eddies whose active central parts are located at $y_{\lambda}=O(\lambda)$. They are only inactive where their wall-normal velocities are blocked by the wall, $y \ll y_{\lambda}$. Their velocities away from the wall therefore scale with $u_{\tau}$, and their wall-parallel components presumably retain the same scaling. The energy in the spectral band $y_{\lambda} / 2 \leqslant \lambda<y_{\lambda}$ is then $O\left(u_{\tau}^{2}\right)$, and since such eddies exist for all $y<y_{\lambda}=O(\lambda)<h$, integration of the spectrum between those limits results in a squared intensity proportional to $\log (h / y)$.

This still implies that the velocity scale for the fluctuations at a fixed $y / h$ is $u_{\tau}$, but when such inactive variables are measured at a fixed $y^{+}$, where $y / h=R e_{\tau}{ }^{-1} y^{+}$, their squared intensities should be proportional $u_{\tau}^{2} \log R e_{\tau}$. For example, that should be true in the buffer layer.

The last conclusion has been tested by several groups over the last decade. Convincing evidence that $u^{\prime+}$ increases in the buffer layer with the Reynolds number was first provided by DeGraaff \& Eaton (2000). They proposed for $u^{\prime 2}$ an empirical 'mixed' scaling with $u_{\tau} U_{c}$, where $U_{c}$ is the free-stream velocity. Since $U_{c}^{+} \sim \log R e_{\tau}+C$, the mixed scaling agrees at very high Reynolds numbers with the logarithmic dependence derived above, although the good agreement at the Reynolds numbers of the experiments may be partly coincidental. A discussion of the bufferlayer spectra of the present channels and of selected experimental results can be found in Jiménez, del Álamo \& Flores (2004) and Hoyas \& Jiménez (2006), who showed explicitly that the wavevectors limiting the spectra separate from each other as the Reynolds number increases.

In fact, the spectral argument implies that the intensities in the buffer layer should be controlled by the size disparity between the largest structures in the outer region and the smallest ones near the wall, rather than by $R e_{\tau}$. This is confirmed by preliminary results by Iwamoto et al. (2006). The width $\Lambda_{z}$ of the largest outer scales differs between boundary layers, Poiseuille, and Couette flows, and they have shown that the near-wall intensities at moderate Reynolds numbers correlate better with $\Lambda_{z}^{+}$ than with $R e_{\tau}$.

\section{Velocity fluctuations}

\subsection{Attached and detached variables in the logarithmic layer}

Before getting into the detailed consideration of the fluctuation profiles, we need to address three questions. The first one is whether the Reynolds numbers of our simulations are high enough to have useful logarithmic layers. The second is whether different types of flows are similar enough above the buffer layer for useful comparisons to be made, and the last one is which variables support attached eddies, and which ones do not.

The answer to the first question is in part a matter of definition. It was shown in the original publications that the simulations have approximately logarithmic mean velocity profiles, specially in the cases of L950 and L2000, but a better test for our purposes is whether there is a range of wall distances in which the length scales increase linearly with $y$. Figure $1(a-c)$ shows premultiplied streamwise spectra of $u$ as 

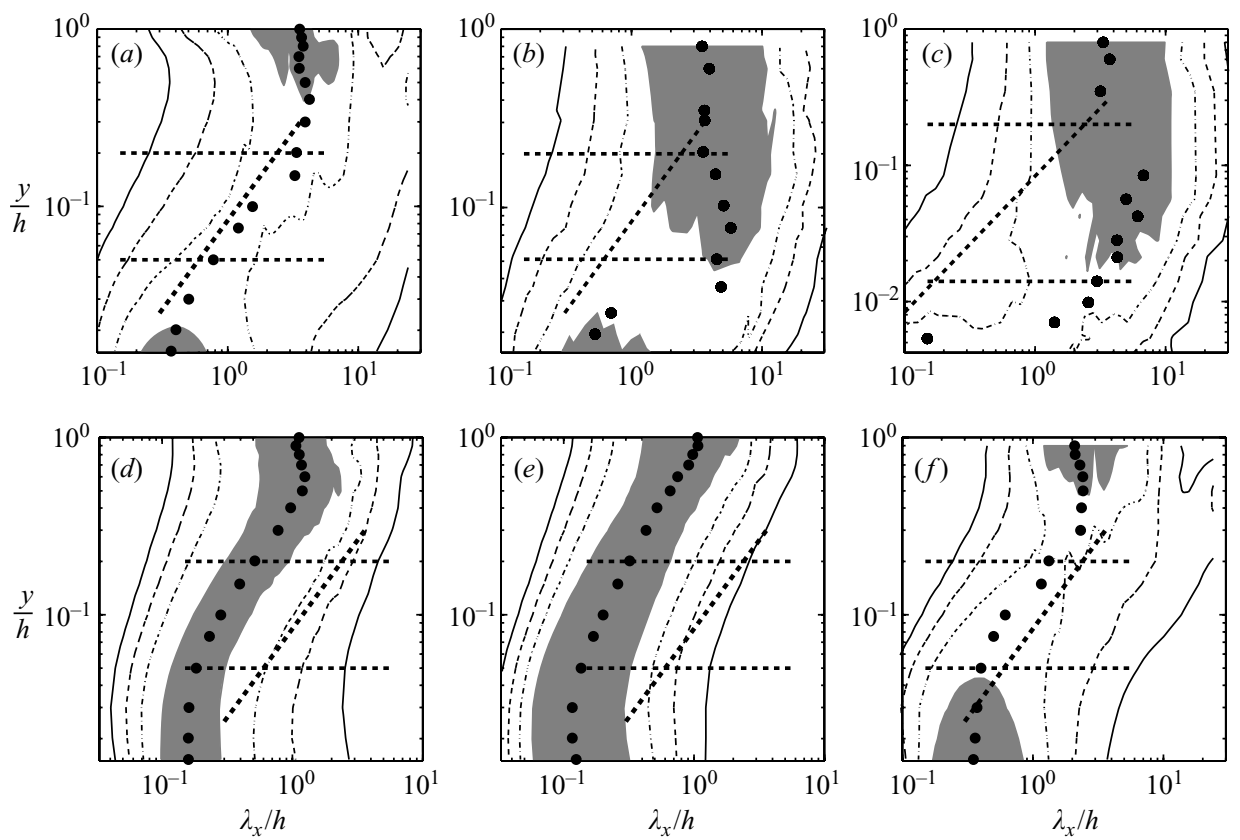

FIGURE 1. $(a-c)$ Normalized premultiplied spectral densities of the streamwise velocity, $\phi_{u и} / u^{\prime 2}$, versus the streamwise wavelength $\lambda_{x}=2 \pi / k_{x}$ and $y / h$. (a) Numerical channel L2000, $(b)$ boundary layer at $R e_{\tau}=1950$, and $(c)$ at $R e_{\tau}=7100$ (Hites 1997). $(d-f)$ Normalized spectral densities in L2000 of $(d)$ the spanwise velocity, $\phi_{w w} / w^{\prime 2},(e)$ the wall-normal velocity, $\phi_{v v} / v^{\prime 2}$, and $(f)$ the shear stress, $\phi_{u v} /\langle u v\rangle$. The dashed horizontal lines are in all cases $y^{+}=100$ and $y / h=0.2$, and the dashed diagonal is $\lambda_{x}=12 y$. The line isocontours in $(a-c)$ are $0.08(0.04) 0.16$; the shaded area is within the isocontour 0.20 . The isocontours in $(d-f)$ are $0.10(0.05) 0.25$. The closed circles mark the maxima of the premultiplied spectra at each height.

functions of the streamwise wavelength and of $y$. That component was chosen in part because it is the one for which most experimental data are available, but also because it is a good example of an 'attached' variable. To approach the second question, case L2000 is compared in the figure with two experimental boundary layers, one at a similar Reynolds number, and another at the highest Reynolds number that we could find. The three figures include the location of the maxima of the premultiplied spectra, as well as a linear trend line. It is clear from the figure that channels have a longer linear scaling region than boundary layers, extending over half a decade for this particular variable in L2000. Note that the two boundary layers in figure $1(b, c)$ have similar spectra, even if their Reynolds numbers are very different, while the two low-Reynolds-number flows in figure $1(a, b)$ have very different spectra, even if their Reynolds numbers are very similar. This underscores the difficulty of comparing even the logarithmic layers of different flows in any but rough terms.

In fact, figure $1(b, c)$ shows that, as already noted by Hites (1997) and by Kunkel (2003), the logarithmic region of boundary layers is contaminated by outer modes at all Reynolds numbers, and that it is difficult to identify a region in which the location of their spectral peaks grows linearly with $y$. If boundary layers have a logarithmic layer in the sense used here, those figures suggest that it only extends up to $y / h \approx 0.05$, while a looser limit of $y / h \lesssim 0.2$ can be used for channels. Note that both limits are lower than those derived elsewhere from the analysis of the mean velocity profiles, 

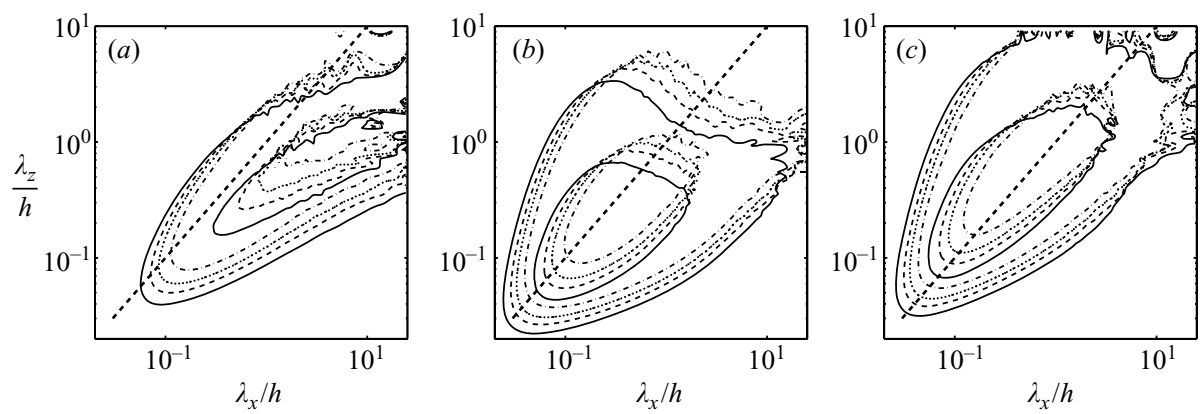

FiguRE 2. Premultiplied two-dimensional spectra for $(a) \phi_{u u},(b) \phi_{v v}$, and $(c) \phi_{w w}$, as functions of $\lambda_{x} / h$ and $\lambda_{z} / h$, at several wall distances in the overlap layer. The spectra are normalized with the local tangential stress, $-\langle u v\rangle=u_{\tau}^{2}(1-y / h)$. Case L2000.,$- y / h=0.1 ;----$, $y / h=0.15 ; \cdots \cdots, y / h=0.2 ;-\ldots, y / h=0.3$. The two contours in each case are 0.125 and 0.625 times the common maximum to the four planes. The dashed diagonal is $\lambda_{x}=\lambda_{z}$.

$y / h=0.15$ for boundary layers (Österlund et al. 2000), and $y / h=0.45$ for channels (Jiménez \& Moser 2007).

It is important to understand that the length scale defined by the spectral maxima in figure 1 is not the integral correlation length. A feature of all the spectra in figure $1(a-c)$ is the presence, from the top of the flow to very near the wall, of eddies with substantial energy at very long wavelengths. The spectrum for the other wall-parallel component, $w$, is shown in figure $1(d)$. Although less clearly than in $\phi_{u u}$, it also has an attached long-wavelength component over the whole flow thickness. What the spectral maximum measures in those cases is the scale of the shorter edge of the energy-containing spectrum, which could be interpreted as the long-wavelength end of the detached isotropic cascade.

Figures $1(e)$ and $1(f)$ show the premultiplied spectrum of $v$ and the cospectrum of the tangential Reynolds stress. The maxima of $\phi_{v v}$ are roughly at the same scales as those of $\phi_{w w}$, and those of $-\phi_{u v}$ are roughly as in $\phi_{u u}$, but both spectra lack most of the energy that the attached variables have in the longer scales. Both $\phi_{v v}$ and $\phi_{u v}$ represent the maximal detached eddies mentioned above, and the spectral lengths used here are their streamwise dimensions.

The differences between attached and detached variables, as well as some of the possible complications, are shown in more detail in figure 2 by the evolution with $y$ of the two-dimensional spectra of the three velocity components. Generally speaking, eddies move to longer and wider wavelengths as they separate from the wall, but they may do so in two different ways. For the detached $\phi_{v v}$ in figure $2(b)$ the spectra have roughly uniform sizes, and their centres 'travel' to larger wavelengths as $y$ increases. For the attached cases in figures $2(a)$ and 2(c) the longest and widest wavelengths have energy across the whole flow. Their long-wavelength limits are approximately independent of the wall distance, and only their short-wavelength edges move to larger scales away from the wall.

Note that variables, such as $\phi_{u u}$ and $\phi_{w w}$, that appear similar from their onedimensional spectra, are actually quite different in the $\left(\lambda_{x}, \lambda_{z}\right)$ plane. For example, the $\phi_{u u}$ spectra are aligned along axes $\lambda_{z}^{2}=y \lambda_{x}$, and migrate parallel to themselves as $y$ increases. They can be interpreted as intersections of the different $y$ planes with long conical eddies tangent to the wall (del Álamo et al. 2004, 2006). On the other hand, the $\phi_{w w}$ and $\phi_{v v}$ spectra are essentially 'equilateral' at all heights, 
$\lambda_{x} \approx \lambda_{z} \approx 3 y$, suggesting that they form part of a more isotropic common transversevelocity structure. However, while the $\phi_{v v}$ spectra collapse approximately in terms of $\lambda / y$, and consequently 'travel' with $y$ in figure $2(b)$, the long- and wide-wavelength ends of $\phi_{w w}$ scale with $h$, at least in our range of Reynolds numbers. The figure suggests that the attached character of $w$ is due more to the width of its structures than to their length. Most of $\phi_{w w}$ is involved in $\langle v w\rangle$ structures, even if it is attached to the wall, but its widest parts do not carry Reynolds stresses because they do not involve $u$. The exception is the long-wavelength tail seen in figure $2(c)$ near $\lambda_{z}=h$. Since there is very little $v$ in that spectral range, that part of $\phi_{w w}$ has to correspond to inactive inactive $\langle u w\rangle$ eddies.

The overall picture is one of 'short' active eddies that carry both $u$ and $v$. Very wide or very long structures still carry some $v$, although not enough to create appreciable tangential stress, but their spanwise or streamwise velocity components, respectively, can still be relatively strong, because continuity requires that $w \sim \lambda_{z} v / y$ or $u \sim \lambda_{x} v / y$.

Although it is not shown explicitly by the representation in figure 2 , the intensities in the cores of the spectra at different heights collapse much better when normalized with the local Reynolds stress $-\langle u v\rangle$ than with $u_{\tau}^{2}$, suggesting that at least part of the dynamics of the active scales is local to the wall distance of their centres.

Note finally that our use of 'attached' and 'detached' is slightly different from that of other groups (e.g. Perry et al. 1986), who would label as 'attached' any eddy whose dimensions are proportional to $y$. From the point of view of energy-containing eddies, the difference is mostly whether the 'maximal' detached eddies are considered as attached or not. Our eddy classes are designed to differentiate spectra whose bandwidths stay constant from those in which they change as a function of $y$. The fluctuation intensities of our detached variables may remain approximately constant as the wall is approached, but the attached ones have to increase, because their spectra grow longer. A better nomenclature for our two classes could perhaps be 'anchored' and 'floating', since we require the former to be large enough to have substantial energy at the wall, but we have preferred to adapt the classical terminology for our purpose.

\subsection{The intensity profiles}

The profiles of the squared intensities for the three velocity components in our simulations are presented in figure 3, scaled with $u_{\tau}^{2}$. The abscissae of the two upper plots are linear, and those of the bottom ones are logarithmic. None of the profiles scales well in the buffer layer within our range of Reynolds numbers, but their behaviours elsewhere are different. Except for a slight drift in the outer layer, the wall-normal component in figure $3(b)$ appears to be following a roughly linear profile that extrapolates to a finite limit of order unity at the wall. The buffer layer would be infinitely thin in the limit of infinite Reynolds number, and the figure suggests that $v^{\prime 2}$ would tend almost everywhere to a non-singular profile.

The spanwise component also drifts slightly far from the wall, but it has a 'hook' near the wall that cannot be easily extrapolated to a finite limit at $y=0$. We saw in the previous section that $w$ is an attached variable that should have a logarithmic intensity profile. Figure $3(d)$ confirms that the near-wall hook is logarithmic, extending from the buffer layer to $y / h \approx 0.3$. Since the limit of a logarithm is singular at $y=0$, this implies that the maximum value of $w^{\prime}$ would grow without bound as the Reynolds number increases. Note that the $v^{\prime 2}$ profiles in figure $3(d)$ have broad maxima, instead of logarithmic layers. 

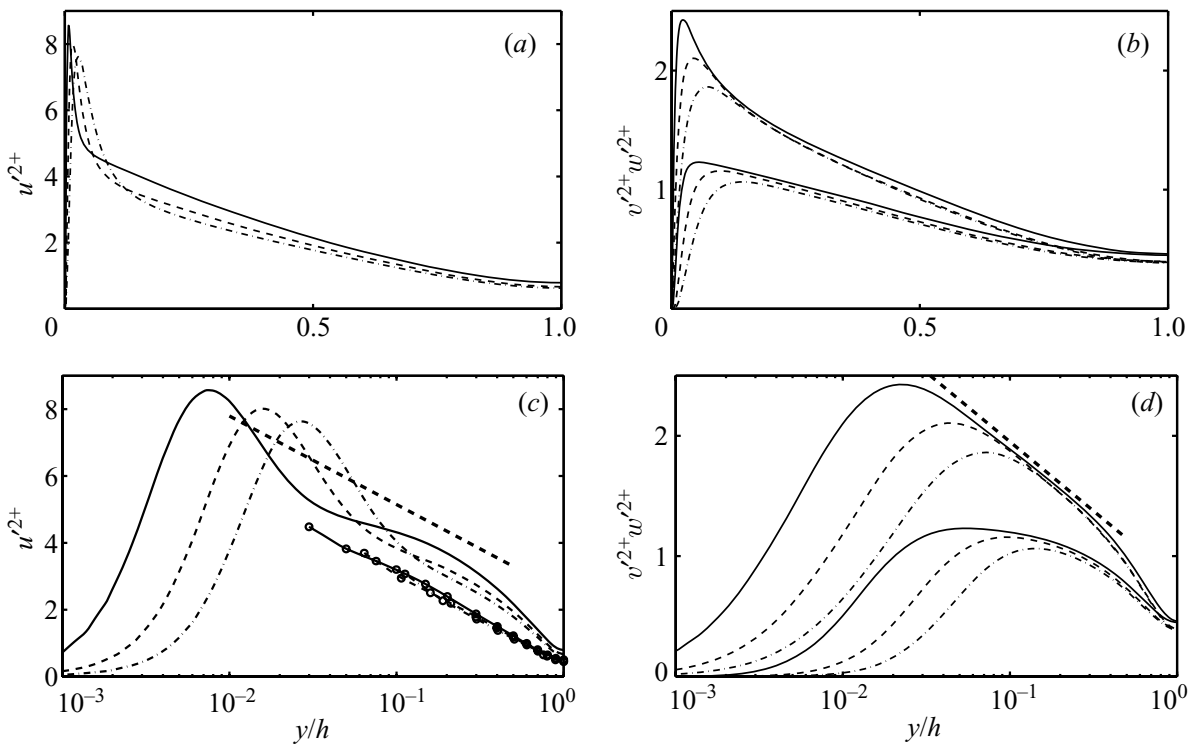

Figure 3. $(a, c)$ Mean-squared profiles of the $u$ fluctuations, for the three numerical channels in table 1. The lines with symbols in $(c)$ are fluctuations with $\lambda_{x}<6 h$ and $\lambda_{z}<h$, above $y^{+}=50$. $(b, d)$ The upper set of curves is $w^{\prime 2}$, and the lower one is $v^{\prime 2}$. The fluctuations are scaled with $u_{\tau}$, and the lines are as in table 1 . The thick dashed lines in the two lower semilogarithmic plots are $u^{\prime 2}=-1.15 \log (y / h)+2.5$ in $(c)$, and $w^{\prime 2}=-0.5 \log (y / h)+0.8$ in $(d)$.

The most complex profiles are those of $u^{\prime 2}$, which do not scale well with $u_{\tau}^{2}$ either near or far from the wall. We saw in $\S 3$ that $\phi_{u u}$ is attached, and that $u^{\prime 2}$ should have a logarithmic profile in the overlap layer. That is harder to spot in figure $4(c)$ than in $w^{\prime 2}$, because the profiles are contaminated with the peaks caused by the streaks of the buffer layer. We have added to figure $3(c)$ a possible fit to the logarithmic slope of the fluctuations The agreement is only moderate, but the Reynolds numbers are low, even in case L2000. Perry et al. (1986) suggested a slightly different fit for the $u^{\prime 2}$ profiles in pipes, and noted that there should be a viscous correction that is not negligible in the lower logarithmic layer. That correction acts in the right direction to improve the fit for the profiles in figure $3(c)$, but it cannot account for the continuous drift of the additive constant of the logarithmic law.

To check the validity over a wider range of Reynolds numbers of the trends just mentioned, figure 4 compares our intensity profiles with a variety of other experimental and numerical data. The streamwise velocity fluctuations in figure 4(a) agree reasonably well with the trend line in figure $3(c)$, although it is also clear in this case that the additive constant is Reynolds-number-dependent. Figure 4(b) displays the behaviour of $u^{\prime 2}$ at $y / h=0.3$ as a function of $R e_{\tau}$, and shows that the drift is not a viscous correction. Viscous effects at such distances from the wall are negligible for all the cases in the figure.

Most of the drift and of the poor logarithmic fit of this velocity component is actually due to the very long and wide eddies found in $u$. The lines with symbols in figure $3(c)$ are intensities computed after subtracting all the large modes identified as 'global' in del Álamo et al. (2004). The results collapse much better than the full fluctuations, and they also follow a much better logarithm. 

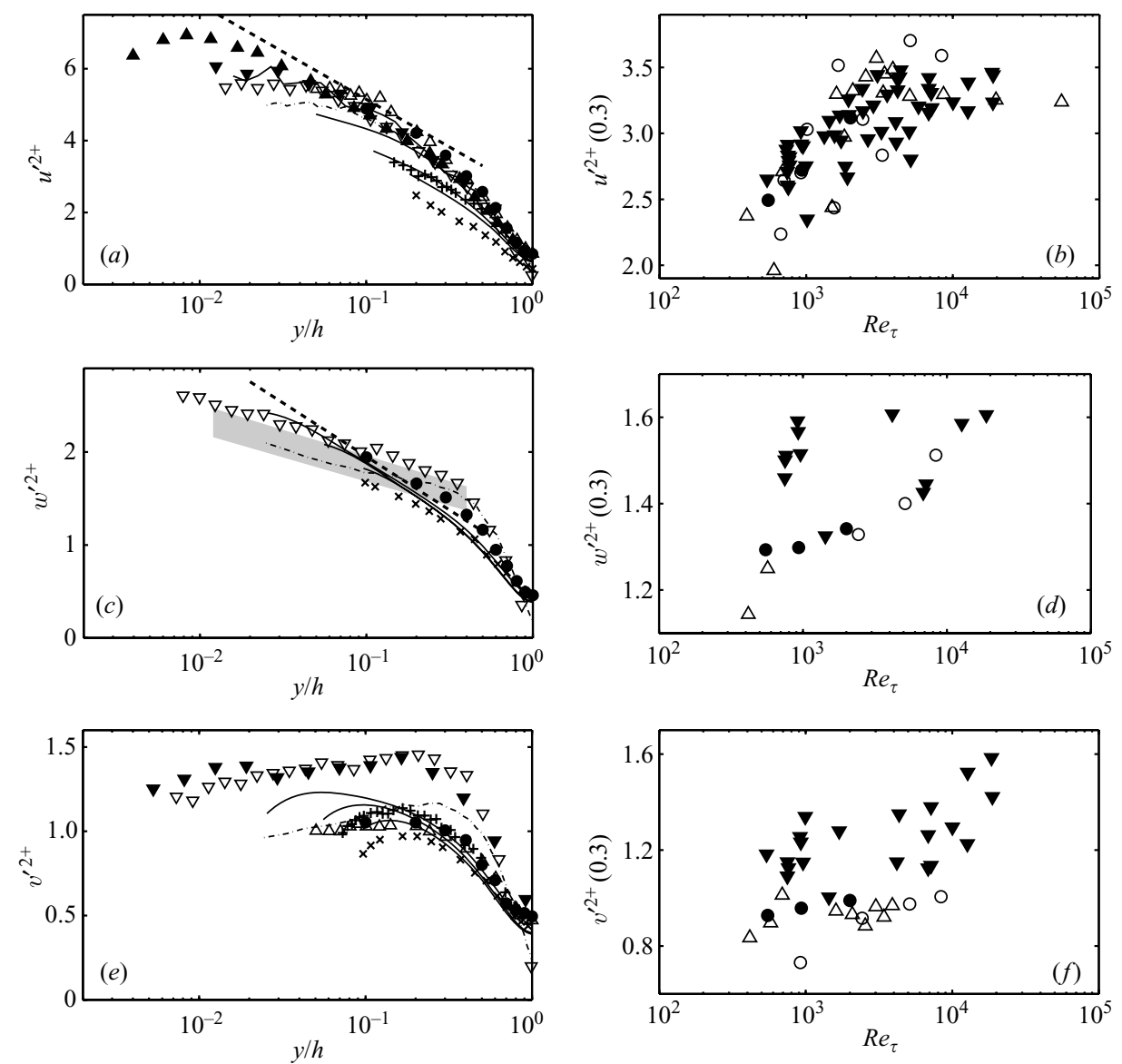

FIgURE 4. $(a, c, e)$ Mean-squared fluctuation profiles, scaled with $u_{\tau}^{2}$, from $y=h$ to the lowest available data point above $y^{+}=100$ in $(a, b)$, and above $y^{+}=50$ in $(c-f)$. $\boldsymbol{\Delta}$, pipe, $R e_{\tau}=10^{5}$ (Morrison et al. 2004); $\boldsymbol{\nabla}$, boundary layer, $R e_{\tau}=10^{4}$ (DeGraaff \& Eaton 2000); $\bullet$, channel, $R e_{\tau}=8400$ (Comte-Bellot 1965); ----, boundary layer, $R e_{\tau}=7120$ (Hites 1997); $\nabla$, boundary layer, $R e_{\tau}=7000$ (Fernholz et al. 1995); - - - boundary layer, $R e_{\tau}=4170$ (Smith 1994); $\triangle$, pipe, $R e_{\tau}=3900$ (Perry et al. 1986); - - present channels, $R e_{\tau}=550-2000$; +, pipe, $R e_{\tau}=690$ (der Toonder 1995). ×, pipe, $R e_{\tau}=568$ (Durst, Jovanovic \& Sender 1995). $(b, d, f)$ Mean-squared fluctuations at $y / h=0.3$, from various sources. $\bigcirc$, channels; $\triangle$, pipes; $\boldsymbol{\nabla}$, boundary layers. The closed circles are the present simulations. $(a, b) u^{\prime 2} .(c, d) w^{\prime 2} .(e, f) v^{\prime 2}$. The dashed straight lines in $(a)$ and $(c)$ are as in figure 3 . The shaded patch in $(c)$ is the range of values given by Fernholz \& Finley (1996) for boundary layers in the range $R e_{\tau}=900-19000$.

The other four panels in figure 4 collect information for the transverse velocity components, $v$ and $w$. As expected from the theoretical arguments, $w^{\prime 2}$ has a logarithmic range below $y / h \approx 0.3$, although the slope of the logarithm in our channels is different from the boundary layers. That is true both for the high-Reynolds-number cases of Fernholz \& Finley (1996) and for the intermediate-Reynolds-number case from Smith (1994). Another boundary layer described by Perry \& Li (1990) at $R e_{\tau}=3700$, not included in the figure, has a logarithmic slope for $w^{\prime 2}$ which is closer to those of the boundary layers than to the channels in figure $4(c)$. The same is true for the highest Reynolds number in the boundary layer simulations of Spalart (1988), whose $R e_{\tau}$ is comparable to that of case L550. 
Inspection of the profiles suggests that the problem is not so much with the logarithmic range itself as with a bulge in the boundary layers that raises the upper end of the logarithmic profiles. The bulge is absent in our channels, but the only higher-Reynolds-number internal flow for which we have data (Comte-Bellot 1965) also has a weak bulge. Its magnitude can be quantified by the value of $w^{\prime 2}$ at a fixed $y / h$, which is given in figure $4(d)$. Comte-Bellot (1965) channels look consistent with ours at the lower Reynolds numbers, and with the boundary layers at the higher ones. This suggests that there are two different effects acting on the bulge. It is present in any flow if the Reynolds number is high enough, but only for external flows at low Reynolds numbers.

A clearer difference between boundary layers and internal flows is the distribution of $v^{\prime}$. The profiles are given in figure 4(e), and show no logarithmic range, as suggested both by the theory and by figure 3 . The values at $y / h=0.3$ are in figure $4(f)$. There is no apparent Reynolds-number drift in pipes and channels, but there is one in the boundary layers. The difference is even clearer in the location of the maximum value of $v^{\prime}$, which is achieved at $y / h \approx 0.2$ in the boundary layers, and at the edge of the buffer layer in the channels. Figure 49 of Fernholz \& Finley (1996) shows a uniform logarithmic growth of the outer $v^{\prime}$ maximum for boundary layers in the range $R e_{\theta}=400$ to $6 \times 10^{5}$, and Jiménez (2004) also found a different behaviour of the maximum $v^{\prime}$ over rough walls in internal and external flows. One the referees has called our attention to an analysis of hot-wire performance by DeGraaff \& Eaton (2000) that may explain the drift in $v^{\prime}$ as an experimental artifact, but their analysis applies equally well to internal and external flows, and cannot explain the differences observed in the figure. The data from that group are included in figures $4(e)$ and $4(f)$, and are consistent with other experiments.

It is tempting to associate the outer maximum of $v^{\prime}$ with the outer bulge of $w^{\prime}$, especially since both variables have similar wall-parallel dimensions. If that were the case, they would represent a structure in the transverse plane, essentially a large streamwise vortex, that forms in boundary layers but not in internal flows at moderate Reynolds number. Apparently, that structure, or perhaps a hierarchy of such structures, reaches deep enough into the flow to modify the logarithmic layer, and could perhaps be associated with the differences between the spectra of boundary layers and channels in figure 1. Unfortunately, the issue can only be resolved either by new simulations of boundary layers at higher Reynolds numbers, or by similar new experiments in pipes or channels.

\section{3. 'Sterile' eddies and the fluctuations of the outer layer}

Besides the possible differences among the logarithmic layers of different flows, the most interesting result up to now is the drift of the outer values in figure $4(b, d, f)$, since we saw in $\S 3$ that the classical theory predicts that the intensities at fixed $y / h$ should scale with $u_{\tau}$.

The evidence for a drift in $v^{\prime}$ and $w^{\prime}$ is limited, because the experimental data for those variables are scarce, and figure $4(d, f)$ could perhaps be discounted as evidence of experimental noise, but the evidence for the $R e_{\tau}$-dependence of $u^{\prime}$ at $y / h=0.3$ in figure $4(b)$ is more persuasive. Similar plots compiled at other wall distances in the range $y / h=0.1-0.5$ produce similar results. A spectral analysis of that range was performed by del Álamo et al. (2004), who concluded that the only possible explanation was that the 'global' modes with $\lambda_{x} / h \gtrsim 6$ scale differently than the shorter ones. That is consistent with the collapse of the intensity profiles in figure 3(c) once those modes are removed. He further concluded that the global modes scale 
with $U_{c}^{2}$, instead of with $u_{\tau}^{2}$, which is conceivable because they are correlated across the whole channel height. That would result in an additive term in $u^{\prime 2}$ proportional to $U_{c}^{2}$, on top of the regular one proportional to $u_{\tau}^{2}$. This is different from the mixed scaling also tentatively proposed by DeGraaff \& Eaton (2000) for this part of the flow, but del Alamo et al. (2004) showed that it is essentially impossible to distinguish empirically between the two behaviours within the range of experimentally accessible Reynolds numbers. In fact, plots like the one in figure $4(b)$ should be treated with care. If that figure had been drawn for $u^{\prime}$, instead of for $u^{\prime 2}$, it would display an equally good logarithmic trend within the experimental scatter.

It should be mentioned at this point that Morrison et al. (2004) have proposed that the trend in figure $4(b)$ is a low-Reynolds-number effect. Their data for the Princeton superpipe are included in the compilations in figures $4(a)$ and $4(b)$, and they level off beyond $R e_{\tau} \approx 3000$. The isolated triangle at $R e_{\tau} \approx 6 \times 10^{4}$ in figure 4(a) belongs to this data set, and there is at least another point beyond $R e_{\tau}=10^{5}$ with roughly the same intensity. The data are very scattered, but Morrison's interpretation would become more plausible if one could disregard the two channels from Comte-Bellot (1965), which are the two highest circles at $R e_{\tau} \approx 10^{4}$ in figure 4(b). The mean velocity profiles of those channels do not agree with the results of later experiments, and they could perhaps be interpreted as meaning that her estimation of $u_{\tau}$ was slightly low. Without those two points, the high-Reynolds-number end of figure 4(b) could very well be levelling at $u^{\prime 2+} \approx 3.2$. The outer-layer profiles in figure 4(a) also appear to converge to some universal shape for high Reynolds numbers, although again scattered.

With this caveat in mind, we can discuss the theoretical possibilities for such growth of the outer fluctuations. The question can be reduced to whether there are 'sterile' eddies that are inactive everywhere, or whose fraction of the tangential Reynolds stress is negligible for the overall momentum balance. Any velocity fluctuation carrying a large fraction of the tangential Reynolds stress is almost sure to scale with $u_{\tau}$, essentially because that is how $u_{\tau}$ is defined, and the argument for a uniform $u_{\tau}$ scaling rests on the assumption that all the eddies are active somewhere.

That is a reasonable assumption, because the source of the turbulent energy that creates the eddies is the Reynolds stress, but it is possible that some eddies may only be active for a short time during their formation, and spend much of their later lives as slowly decaying sterile objects. If that were the case, their velocities would be unconstrained by the classical argument, and could scale arbitrarily. For example, one could imagine that structures of $u$ are born as active eddies, but that their $v$ component decays soon afterwards. They would then remain for some time contributing to $u^{\prime 2}$, but not to $\langle u v\rangle$, while new active structures would have to be created to satisfy the momentum balance. The new structures would also contribute to $u^{\prime 2}$, and the total streamwise intensity would be determined by the decay rate of the sterile structures, rather than by the tangential stress. The same argument would apply to the inactive parts of active eddies if they could be assumed to decouple from their original cores.

The obvious location for possible sterile eddies are the very long global modes mentioned above. The structures of $v$ cannot be very long or very wide, because they are blocked by the wall, and since $v$ is needed to generate the Reynolds stress, the $\phi_{u v}$ cospectrum could be shorter than $\phi_{u u}$. The scaling of any $u$ structure longer than the cospectrum would be unconstrained. 

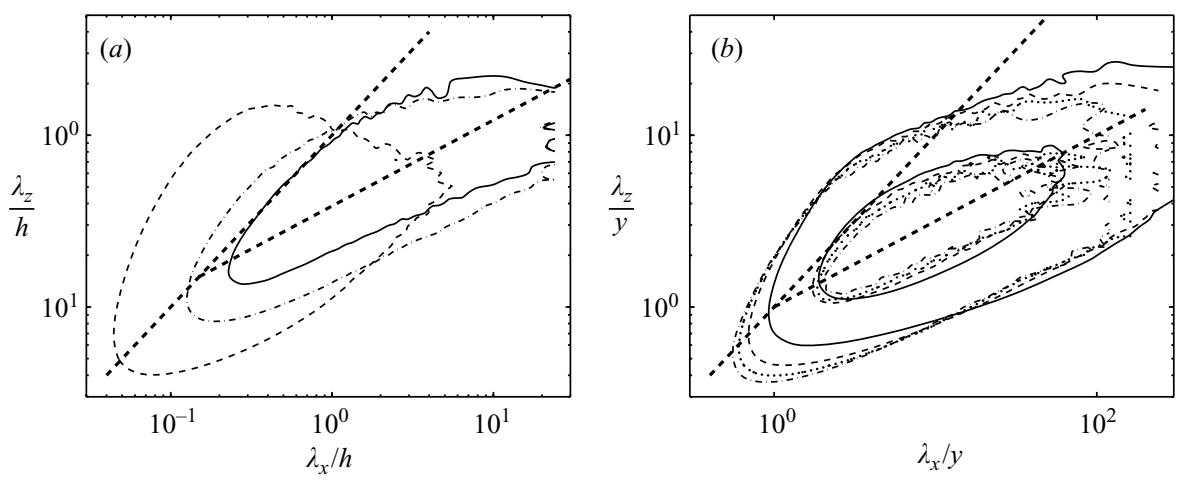

Figure 5. (a) Two-dimensional spectra versus the wavelengths. Case L2000 at $y / h=0.15$. ,$- \phi_{u u} ;----, \phi_{v v} ;---,-\phi_{u v}$. Contours are 0.125 times the maximum for each spectrum. (b) Two-dimensional cospectra $\phi_{u v} /\langle u v\rangle$, versus the wavelengths normalized with the wall distance. Case L2000.,$- y / h=0.05 ;----, y / h=0.1 ; \cdots \cdots, . \cdots, y=0.15 ;-.-, y / h=0.2$. The two contours in each spectrum are 0.125 and 0.625 times the global maximum over the four cases. The steeper dashed diagonal in both figures is $\lambda_{x}=\lambda_{z}$, and the lower dashed line is $\lambda_{z}^{2}=y \lambda_{x}$ (del Álamo et al. 2004).

A problem with this argument is that the cospectra are experimentally known to be very long, even close to the wall. This is seen in figure 5(a), which shows that both $\phi_{u u}$ and $\phi_{u v}$ in the logarithmic layer reach the end of the computational box, $L_{x} / h=8 \pi$. Guala, Hommema \& Adrian (2006) have shown that the very long scales, defined by them in a pipe as $\lambda_{x} \gtrsim 8 h$, carry both a substantial percentage of the energy and of the tangential Reynolds stress, and Balakumar \& Adrian (2007) have done the same for channels and for boundary layers.

The question of the length of the cospectrum can, however, be answered with some assurance. At the Reynolds numbers of the computational channels, the cores of the cospectra scale well both with the total tangential stress, $\langle u v\rangle \approx-u_{\tau}^{2}(1-y / h)$, and with $\lambda / y$. This is shown in figure $5(b)$, which presents four two-dimensional cospectra of L2000 in the range $y / h=0.05\left(y^{+}=100\right)$ to $y / h=0.2$. The agreement of the highest isocontours is very good, showing that the size of the cospectrum scales with $y$. If, for example, it scaled with $h$, it would show clearly over this factor of four in wall distance. The lower isocontours lengthen slightly for short wavelengths, but these are the structures of the quasi-isotropic Kolmogorov energy cascade, and they contain very little tangential stress. The long-wavelength ends of the lower isocontours also drift in the figure proportionally to $h$, but that limit is the length of the computational box, which we have seen to be too short to settle this particular question.

Figures $6(a)$ and $6(b)$ collect $\phi_{v v}$ and $\phi_{u v}$ from several high-Reynolds-number flows, including the atmospheric boundary layer (ABL). This allows us to use a wide range of $y / h$ while staying above the buffer layer, and to distinguish scalings with $y$ from those with $h$. If we take the depth of the ABL to be $h \approx 200 \mathrm{~m}$, the figure spans a factor of about 30 in $y / h$. Under those circumstances, the collapse of both $\phi_{v v}$ and $\phi_{u v}$ is extremely good, although both quantities reach surprising lengths. The $v$ structures are centred around $\lambda_{x} \approx 2 y$, but they still have substantial energy at $\lambda_{x} / y=100$. The $\phi_{u v}$ cospectra centre at $\lambda_{x} \approx 20 y$, but they decay faster, and extend only slightly beyond $\phi_{v v}$. For the $1 \mathrm{~m}$ level of the ABLs in figure $5(b-d)$, the longest limits of both quantities are $\lambda_{x} \approx h$, but they would be $\lambda_{x} \approx 20 h$ for $y / h \approx 0.1$. These are very long structures, but they are nevertheless multiples of $y$, and they could 

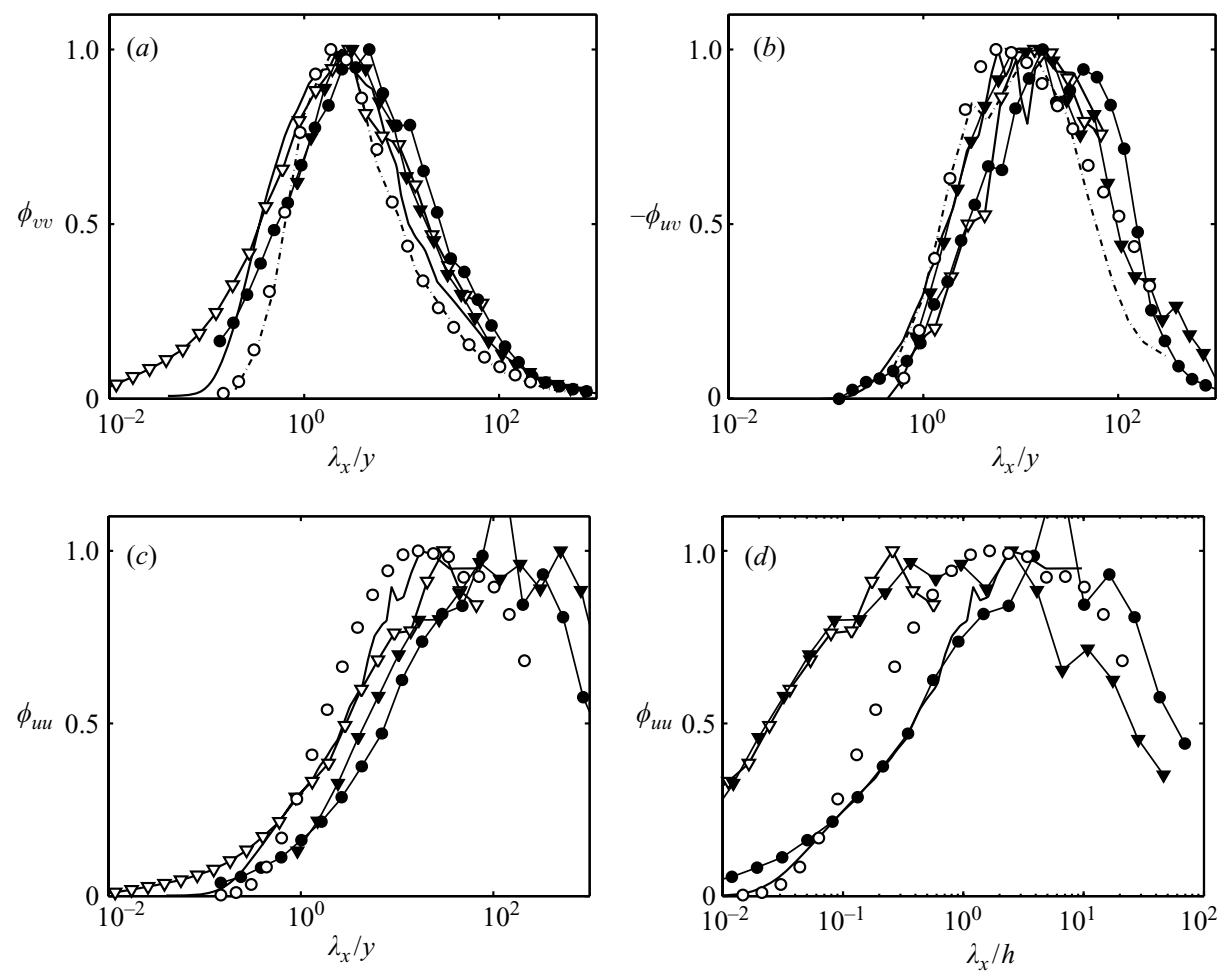

FIGURE 6. One-dimensional premultiplied spectra of $(a)$ wall-normal velocity versus $\lambda_{x} / y,(b)$ cospectrum $-\phi_{u v}$ versus $\lambda_{x} / y,(c)$ streamwise velocity versus $\lambda_{x} / y,(d)$ streamwise velocity versus $\lambda_{x} / h$, in the logarithmic layers of experimental and atmospheric flows. The spectra are normalized to unit maxima, because of the uncertainty in the actual Reynolds stresses. $\nabla$, ABL, $y=1 \mathrm{~m}, u_{\tau}=0.65 \mathrm{~m} \mathrm{~s}^{-1}, h=200 \mathrm{~m}$ (Hoxey \& Richards 1992); $\bullet$, same case $y=10 \mathrm{~m}$; $\nabla$, ABL, $y=1.7 \mathrm{~m}, u_{\tau}=0.24 \mathrm{~m} \mathrm{~s}^{-1}, h=200 \mathrm{~m}$ (Antonia \& Pearson 1999); — , boundary layer, $y / h=0.14, R e_{\tau}=7000$ (Carlier 2001); - - , pipe, $y / h=0.12, R e_{\tau}=2100$ (Lawn 1971); $\mathrm{O}$, channel L2000, $y / h=0.1$. Both ABLs are fully rough.

be made shorter than any of the $O(h)$ structures in the $\phi_{u u}$ spectra in figure 1 by letting $y / h$ be small enough at sufficiently high $R e_{\tau}$. In that limit, there will always be $u$ structures long enough to be inactive. The $\phi_{u u}$ spectra of the flows in figures $6(a)$ and $6(b)$ are given in figure 6(c), and they are all too long to close within the available range of wavelengths. All the $u$ structures to the right of $\lambda_{x} / y \approx 100$ can be considered as essentially inactive.

Figure $6(d)$ shows $\phi_{u u}$ against the wavelengths scaled with the boundary layer thickness. At least at these distances from the wall, the longest scales are of the order of $20-40 h$, which agrees with the absolute maximum lengths found by del Álamo et al. (2004) at somewhat lower Reynolds numbers. It is also consistent with the spectra in figure 1 . We are not aware of tangential-stress cospectra in the outer layer at really high Reynolds numbers, but if we take figures $1(f)$ or $6(b)$ as guides, the longest cospectra peak at $\lambda_{x} \approx 2 h$ near the top of the logarithmic layer, and are essentially zero beyond $\lambda_{x} \approx 20 h$ at all heights. There is therefore a range of $\phi_{u u}$ that is globally inactive, and whose intensity is not required to scale with $u_{\tau}$. As we have already mentioned, del Álamo et al. (2004) found that those modes scale best with the centreline velocity, $U_{c}^{2}$, suggesting that the growing trend of $u^{\prime}$ away from the wall would extend indefinitely at high Reynolds numbers. 

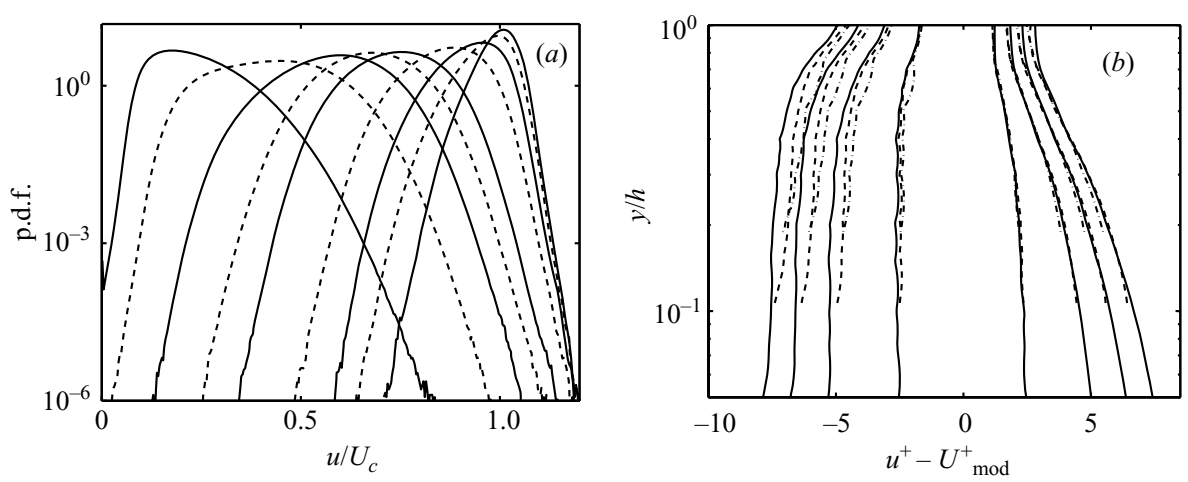

Figure 7. (a) Probability density functions of the streamwise velocity, normalized with the centreline velocity. Case L2000. From left to right, $y^{+}=6,14,40,100 ; y / h=0.1,0.3,0.5,0.7$, 1. (b) Probability density functions of the streamwise velocity fluctuations with respect to the local mode, in wall units, as a function of the wall distance above $y^{+}=70$. Data from the numerical channels. Lines as in table 1 . The contours are logarithmically spaced by factors of 10 , from $10^{-1}$.

\subsection{The probability density functions}

Further information on the behaviour of the velocity fluctuations is obtained from their probability density functions (p.d.f.s). It is known that the skewness coefficient of the streamwise velocity changes sign from positive near the wall to negative above the buffer layer (Nakagawa \& Nezu 1977). In fact, the third-order moment of $u$ changes sign above the buffer layer, and levels around $\left\langle u^{3}\right\rangle^{+} \approx-1.5$ until it rises again near the top of the flow (Fernholz \& Finley 1996). The p.d.f.s responsible for those changes are shown for case L2000 in figure 7(a). It is interesting that even within the viscous sublayer $\left(y^{+}=6\right)$ there are velocity incursions which are of the order of the centreline velocity, and that in the core of the buffer layer $\left(y^{+}=40\right)$ the velocity can exceed $U_{c}$. The p.d.f.s in the logarithmic range $\left(y^{+}=40-200\right)$ are roughly symmetric in this particular case, but above that level they become skewed in the opposite direction, as their high-velocity tail becomes limited by the maximum instantaneous velocity in the channel $\left(\approx 1.2 U_{c}\right)$. It is interesting that, while the high velocities reach very near the wall, the lowest velocities away from the wall are more moderate.

The evolution with the Reynolds number is shown in figure $7(b)$, where the p.d.f.s of the three numerical channels are shown normalized with $u_{\tau}$, and centred around their local modal values. The mode is a better reference than the mean, because it represents the most probable velocity and is not affected by the behaviour of the probability tails. What the figure shows is that, for a fixed $y / h$, the high-velocity tail of the p.d.f. remains essentially independent of the Reynolds number, while the low-velocity tail gets wider with increasing $R e_{\tau}$. The simplest interpretation is that the flow at a given height collects fluid from all over the channel. The difference between the velocity at a given $y / h$ and the maximum velocity at the centreline is independent of the Reynolds number, because so is the velocity defect. The flow has no way of widening its high-velocity probability tail. On the other hand, the velocity difference with the wall increases roughly as $\log R e_{\tau}$. As the Reynolds number increases, the flow has a deeper reservoir from which to draw low-velocity fluid, its low-velocity tail widens, and $u^{\prime}$ increases. 

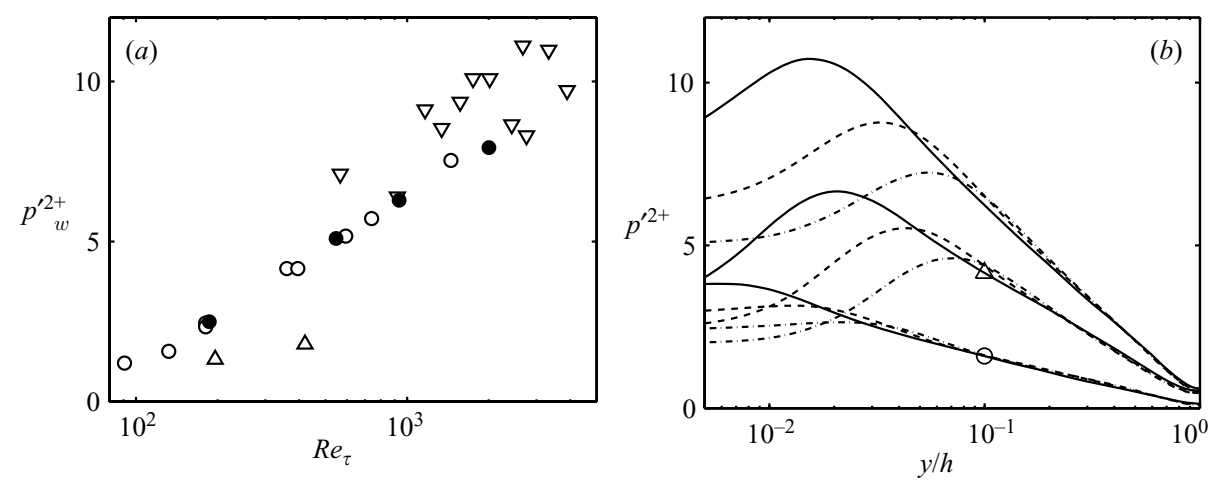

Figure 8. (a) Mean-squared pressure fluctuations at the wall as a function of $R e_{\tau}$, from various sources. $\bigcirc$, channels; $\triangle$, pipes; $\nabla$, boundary layers. The closed circles are the present simulations. (b) Fluctuation profiles. Lines as in table 1. Lines without symbols are the full pressure. $\bigcirc$, fast pressure; $\triangle$, slow pressure.

\section{Pressure fluctuations}

Pressure fluctuations are of special interest because there is little experimental information on them. On a dimensional basis, pressure should scale with $u_{\tau}^{2}$, but the pressure fluctuations at the wall increase strongly with the Reynolds number. The compilation in figure 8(a), taken mostly from Hu, Morley \& Sandham (2006), shows a roughly logarithmic increase of $p_{w}^{\prime 2+}$ in the range $R e_{\tau}=100-4000$, although again with some scatter. Pressure is also special in that fluctuations cannot be defined unambiguously for an incompressible flow. The equations of motion are insensitive to a spatially uniform, temporally variable additive pressure, which would, however, appear as part of the fluctuations. It is usually possible to refer measurements to some point defined as a constant, but the definition varies among experiments, and complicates comparisons. It may not be a coincidence, for example, that the lowest fluctuations in figure 8(a) correspond to a pipe in which the experimenters took special care to remove low-frequency components and spurious vibrations (Lauchle \& Daniels 1987).

The ambiguity is specially serious in internal flows, because they are driven by a pressure gradient that has to be subtracted before fluctuations can be defined. Otherwise, spatial averages would be very different from temporal ones, and even the latter may be contaminated by low-frequency oscillations due, for example, to variations in the flow rate. We have adopted for our numerical channels the convention of neglecting the instantaneous uniform pressure gradient used to maintain a constant mass flux. Even in our large computational boxes, that gradient varies slightly with time, and taking it into account would have formally led to infinite fluctuations.

These ambiguities raise the question of whether pressure fluctuations are really important, or whether we should just worry about the statistics of the pressure gradient, which is the quantity in the equations of motions. In fact, although the Navier-Stokes equations contain only pressure gradients, they are equations for accelerations, while the variables of interest are usually velocities. The latter are integrals of the former, and the relevant quantities are the pressure differences, which are the integrals of the gradients. Pressure is relevant in spite of the ambiguity of its mean value for the same reason that velocities are relevant in spite of Galilean invariance. A trivial example is the averaged wall-normal momentum equation for a 

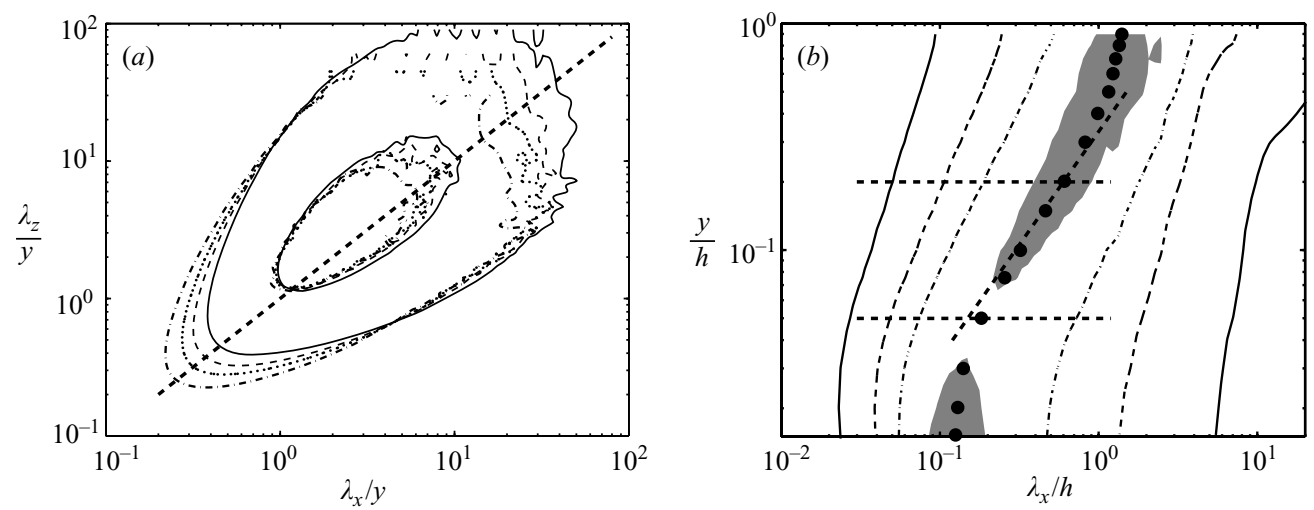

FiguRE 9. Spectral density functions for the pressure fluctuations in case L2000. (a) As a function of $\lambda_{x} / y$ and $\lambda_{z} / y . \longrightarrow, y / h=0.1 ;-\cdots, y / h=0.15 ; \cdots \cdots, y, y / h=0.2 ;-\cdot-$, $y / h=0.3$. The two contours in each spectrum are $\phi_{p p} /\langle u v\rangle^{2}=0.125$ and 0.625 times the common maximum of the four cases. (b) As a function of $\lambda_{x} / h$ and $y / h$. Contours are $\phi_{p p} / p^{\prime 2}=0.03(0.09) 0.3$. The dashed horizontal limits, diagonal and closed circles, are as in figure 1 .

parallel shear flow, whose useful form is

$$
\langle p\rangle+\left\langle v^{2}\right\rangle=\text { constant, }
$$

even if what the Navier-Stokes equations state is that the mean wall-normal pressure gradient is equal to the mean wall-normal acceleration.

The logarithmic behaviour of the pressure fluctuations across the logarithmic layer of our channels is displayed in figure $8(b)$, and extends from the centre of the channel to $y^{+} \approx 40$. The 'fast' and 'slow' components given separately in figure $8(b)$ are defined in Kim (1989), and they are of interest because they tend to be modelled separately by the RANS community. Briefly, they refer to different terms on the right-hand side of the Poisson equation used to compute the pressure fluctuations. For a incompressible flow,

$$
\nabla^{2} p=-2 \partial_{i} U_{j} \partial_{j} u_{i}-\partial_{i} u_{j} \partial_{j} u_{i} \equiv R .
$$

The first term on the right-hand side is linear in the fluctuations, and gives rise to the 'fast' component. The second one is fully nonlinear, and is the origin of the 'slow' term. Both pressures are comparable everywhere in figure $8(b)$. They scale as well as the full pressure in the outer region, and as badly in the buffer layer, and they both have equally good logarithmic ranges. In wall-bounded flows, (5.2) is usually integrated with homogeneous Neumann boundary conditions at the wall, while the real boundary conditions have a non-zero viscous contribution. The difference is the 'Stokes' pressure (Kim 1989), but in the channels it is negligible everywhere at these Reynolds numbers. For the rest of this section we will only concern ourselves with the total pressure fluctuations.

The pressure spectra are given in figure 9. They are closer to $\lambda_{x}=\lambda_{z}$ than other quantities discussed in this paper, corresponding to features that are roughly 'equilateral' in the wall-parallel plane. Their peaks in the logarithmic layer are located roughly at the same location as those for $\phi_{w w}$. Their long-wavelength limits scale with $h$, but pressure lacks the tail associated with $\langle u w\rangle$ structures in $\phi_{w w}$. It is interesting that, once again, the magnitude of the central peak scales much better with the local Reynolds stress than with $u_{\tau}$. Note that $\langle u v\rangle^{2}$ varies in this case by $60 \%$ among the 
different planes in figure $9(a)$, and that any scaling error would show immediately in the figure.

Although that scaling does not extend much beyond the logarithmic region, it suggests that, although it is generally considered to be a global quantity because it can be derived as the solution of an elliptic equation such as (5.2), the pressure eddies near the spectral peak are fairly local to each wall distance. That agrees with the analysis of its governing equation. If we denote by $\widehat{p}\left(k_{x}, k_{z}\right)$ the Fourier transform of $p$, and use a similar notation for other variables, we can write the solution to (5.2) in terms of its right-hand side $R$, and of a Green's function $G$, as

$$
\widehat{p}(y)=\int_{0}^{2 h} \widehat{R}\left(y^{\prime}\right) G\left(y, y^{\prime}\right) \mathrm{d} y^{\prime},
$$

where $G$ is a tent-like combination of $\exp \left( \pm y^{\prime} / \lambda\right)$, centred at $y$, and $\lambda^{2}=\lambda_{x}^{2}+\lambda_{z}^{2}$. Since it is clear from figure 9 that the interesting spectral range is $\lambda_{x} \approx \lambda_{z}$, we will use in the following $\lambda$ and $k=2 \pi / \lambda$ as interchangeable with any of the wavelengths or wavenumbers. The pressure spectrum can be written as

$$
\left\langle|\widehat{p}|^{2}\right\rangle(y)=\int_{0}^{2 h} \int_{0}^{2 h} C_{R R}\left(y^{\prime}, y^{\prime \prime}\right) G\left(y, y^{\prime}\right) G\left(y, y^{\prime \prime}\right) \mathrm{d} y^{\prime} \mathrm{d} y^{\prime \prime},
$$

where $C_{R R}\left(y^{\prime}, y^{\prime \prime}\right)$ is the correlation of $\widehat{R}$ at two wall distances, and $G\left(y, y^{\prime}\right) G\left(y, y^{\prime \prime}\right)$ is a two-dimensional Green's function. The number of samples in our simulations is not enough to compute $C_{R R}$ with a reasonable signal-to-noise ratio, but two conclusions from the limited results at hand are that the width in $y^{\prime}$ of $C_{R R}$ is approximately $10-20 \eta$ at all wavelengths, where $\eta$ is the Kolmogorov viscous scale, and that the spectrum, $\phi_{R R}=k^{2} C_{R R}(y, y)$, is at roughly the same wavelengths as the vorticities. Since $R$ is a combination of velocity gradients, both observations make sense.

There are two interesting limits to (5.4). For $\lambda \leqslant h$ and $y / h=O(1)$, the twodimensional Green's function has width $\lambda$, and $G^{2}(y, y) \approx \lambda^{2}$. The integrand of (5.4) is non-zero over an area $O(\lambda \eta)$ in the neighbourhood of $y^{\prime}=y^{\prime \prime}=y$, and the integral can be manipulated into

$$
\phi_{p p}\left(y ; k_{x}, k_{z}\right) \approx k^{2}\left\langle|\widehat{p}|^{2}\right\rangle \approx \lambda^{3} \eta \phi_{R R}(y),
$$

which is consistent with the local nature of pressure at small wavelengths.

On the other hand, when $\lambda \gg h$ the two-dimensional Green's function is essentially constant, equal to $G^{2}\left(y^{\prime}, y^{\prime \prime}\right) \approx \lambda^{4} / h^{2}$ everywhere. The integrand in (5.4) is still concentrated in a narrow band $y^{\prime \prime}=y^{\prime} \pm O(\eta)$, but the integral now extends over all $y^{\prime}$. It follows that

$$
\phi_{p p}\left(y ; k_{x}, k_{z}\right) \approx \frac{\lambda^{4} \eta}{h^{2}} \int_{0}^{2 h} \phi_{R R}\left(y^{\prime}\right) \mathrm{d} y^{\prime},
$$

whose main message is that $\phi_{p p}$ is independent of $y$ for large wavelengths.

The behaviour with the wall distance of the one-dimensional pressure spectra is given in figure $9(b)$. As should be expected from the previous discussion, there is a central core whose wavelength increases linearly with $y$, and a long-wavelength component that reaches a constant wavelength at all wall distances. In the same way as in our discussion of $\phi_{u u}$ and $\phi_{w w}$, this justifies the logarithmic profile of the fluctuations.

It is interesting that the pressure is a relatively 'short' quantity, whose spectrum does not extend much beyond $\lambda_{x} \approx 5 h$, even if (5.6) suggests that it should increase 
with $\lambda$. The velocity gradients, and therefore the right-hand side $R$, reside at lengths of the order of $\eta$ and have very little energy at large scales, but when the $\lambda^{4}$ weighting introduced by the Laplacian is taken into account, it turns out that most of the weight should be in the upper right-hand corner of the wavelength plane. However, even if the effective spectrum is most intense there, the wall-normal structure of the correlation function is such that its contribution to the pressure cancels upon convolution, apparently fortuitously. A moment of reflection shows that the reason is that these wide and long vorticity structures are vortex sheets that, contrary to vortex filaments, are parallel flows in which the accelerations are automatically zero. They therefore do not produce pressure fluctuations. Long vortex filaments, with lengths of the order of several channel widths and thickness of the order of the Kolmogorov scale, would create localized small-scale pressure fluctuations, but the spectra of $R$ and of the vorticities show that there are not enough of them in the flow to contribute appreciably to the pressure. Note that, because of the lack of very large scales, which are the ones for which the velocity has been shown to scale anomalously, the pressure fluctuations away from the wall scale very well with $u_{\tau}^{2}$.

\section{Summary and conclusions}

We have reviewed the behaviour of the velocity and pressure fluctuations in the logarithmic and outer layers of turbulent wall-bounded flows, using mostly data from numerical simulations of turbulent channels with $R e_{\tau} \lesssim 2000$, but also experimental and atmospheric data at comparable or higher Reynolds numbers. Our results include the fluctuation intensity profiles, their decomposition in terms of spectral energy densities, and the velocity probability density functions.

As expected from previous experimental evidence, and as initially proposed by Townsend (1976), the intensities of the two wall-parallel velocity components have logarithmic profiles that extend across the logarithmic layer of the mean velocity. This implies that their fluctuations in the buffer layer increase with $\log \left(R e_{\tau}\right)$ when expressed in wall units, which is approximately the same as the mixed scaling proposed by DeGraaff \& Eaton (2000). Both components have spectra that are anchored to the wall, and that become longer and wider as they approach it. Their long-wavelength limits scale with $h$ at essentially all wall distances. The streamwise velocity spectra are aligned along $\lambda_{z}^{2} \approx y \lambda_{x}$, which can be interpreted as representing conical velocity structures. The spectra of the spanwise velocity, $\phi_{w w}$, are closer to $\lambda_{x} \approx \lambda_{z} \approx 3 y$, which are the dimensions of the velocity ejections identified by del Álamo et al. (2004).

The spectra of $v$ are detached, in the sense that, although their maxima are located at the same wavelengths as the cores of $\phi_{w w}$, they have no large-scale component at the wall. Consequently, the $v^{\prime 2}$ profile has no logarithmic range. However, the $\phi_{v v}$ structures are surprisingly long, $\lambda_{x} / y=O(100)$, corresponding to several flow thicknesses at the top of the logarithmic layer. The same is true of the tangential Reynolds stress cospectrum, $\phi_{u v}$ which, however, also scales ultimately with $\lambda_{x} \sim y$.

The spectra of $w$ are shorter than those of $u$ but, at the Reynolds numbers of our simulations, they have a weak tail at the same long wavelengths as $\phi_{u u}$. There are few experimental data for this tail at higher Reynolds numbers, but continuity requires that it should be there. Since the spectra of the wall-normal velocity are centred at $\lambda_{x} \approx y$, with very little energy at $\lambda_{x}=O(h)$, the only velocity component that can compensate for $\partial_{x} u$ is $w$. Hutchins \& Marusic (2007) have identified the long 
$u$ structures as meandering jets of essentially infinite length, where the streamwise wavelength corresponds to the meanders. The long $\phi_{w w}$ component would correspond to the meandering motion.

Pressure has wall-attached spectra, and a very well-developed logarithmic range in the fluctuation profile. As such, its fluctuations at the wall increase strongly as $\log R e_{\tau}$. This wall-attached character can be derived theoretically from the form of the Green's function of the pressure Poisson equation. It provides a justification for the origin of the attached velocity eddies, since Bradshaw (1967) observed that the pressure perturbations reaching the wall should create tangential velocity perturbations at similar scales. The maximum length of the pressure spectrum is shorter than any of the attached velocity components, $\lambda_{x} \lesssim 5 h$, with no fluctuations due to the very long scales of $u$ and $w$. We have given arguments for why that should be so, based on the geometry of the large-scale velocity structures. Note that, while all these results are strongly supportive of the attached-eddy scenario of Townsend (1976), most of them are independent of any specific model for the geometry of the eddies.

Another interesting observation is that most of the spectra in the logarithmic layer scale better with the local Reynolds stress $\langle u v\rangle$ than with the friction velocity, suggesting that an important part of the dynamics of that region is local to each wall distance. This agrees with numerical evidence that perturbing the dynamics of the wall leaves the structures of the logarithmic and outer regions unaffected (Flores \& Jiménez 2006; Flores, Jiménez \& del Álamo 2007).

Above the logarithmic layer, the intensity of the streamwise velocity fluctuations at fixed $y / h$ increases with the Reynolds number, at least in the range $R e_{\tau} \approx 5000$ for which experimental data are available from more than one source. In the simulations, the drift disappears when the very large scales are removed, suggesting that they are the ones responsible for it. We have argued that those eddies that do not carry Reynolds stresses are not constrained to scale with $u_{\tau}$ by the usual arguments. The probability density function of the velocity suggests that the increase of $u^{\prime}$ is due to stronger low-velocity ejections coming from the wall, rather than to stronger sweeps, in agreement with previous evidence that the intensity of the large eddies scales with the centreline velocity.

The situation for the transverse velocity components is more confused, specially because substantial differences were found between the present channels and the experimental boundary layers used for comparisons at higher Reynolds numbers. For example, we saw in figure 1 that the outer $\phi_{u u}$ structures penetrate much closer to the wall in boundary layers than in channels, suggesting that the effective logarithmic layer in the former is thinner $(y / h \lesssim 0.05)$ than in the latter $(y / h \lesssim 0.2)$. Note, however, that figure 1 compares spatial spectra for the simulation with temporal ones for the experiments, and that the Taylor approximation may give misleading results for very long wavelengths.

There are outer $\phi_{v v}$ and $\phi_{w w}$ structures in boundary layers that are not present in pipes and channels, and similar outer-layer structures seem to exist in both internal and external flows at high Reynolds numbers. The intensities of those structures do not scale with $u_{\tau}$, and they are responsible for the failure of the classical scaling of $v^{\prime}$ and $w^{\prime}$ in the outer layer. They penetrate close to the wall, and seem to be responsible for the differences between the logarithmic slopes of the $w^{\prime 2}$ profiles in boundary layers and channels. The resolution of these ambiguities would require either new simulations of boundary layers at higher Reynolds numbers, or similar new experiments in pipes or channels. 
This work was supported in part by the CICYT contract TRA2006-08226, and by the EU FP6 Wallturb Strep AST4-CT-2005-516008. The computations of the new statistics were made possible by a generous grant of computer time from the Barcelona Supercomputing Centre, and by the equally generous collaboration of the Port d'Informació Científica (PIC), who lent their mass storage facilities to archive the original raw data, and who hosted our postprocessing computers. SH was supported in part by the Spanish Ministry of Education and Science, under the Juan de la Cierva program. Special thanks are due to Mr G. Caniot, who ran the pressure statistics during a study stage supported by the EU FP5 Training and Mobility Network HPRN-CT-2002-00300. We are grateful to Messrs Antonia, Carlier, Fernholz, DeGraaff, Hoxey, Marusic and Nagib for providing us with electronic copies of their original data.

\section{REFERENCES}

AGARD 1998 A selection of test cases for the validation of large-eddy simulations of turbulent flows. Advisory report. 345. http://torroja.dmt.upm.es/ftp/AGARD.

DEl Álamo, J. C. \& JimÉnez, J. 2003 Spectra of very large anisotropic scales in turbulent channels. Phys. Fluids 15, L41-L44.

del Álamo, J. C., Jiménez, J., Zandonade, P. \& Moser, R. D. 2004 Scaling of the energy spectra of turbulent channels. J. Fluid Mech. 500, 135-144.

del Álamo, J. C., Jiménez, J., Zandonade, P. \& Moser, R. D. 2006 Self-similar vortex clusters in the logarithmic region. J. Fluid Mech. 561, 329-358.

Antonia, R. A. \& Pearson, B. R. 1999 Low-order velocity structure functions in relatively high Reynolds number turbulence. Europhys. Lett. 48, 163-169.

Balakumar, B. J. \& Adrian, R. J. 2007 Large- and very-large-scale motions in channel and boundary-layer flow. Phil. Trans. R. Soc. Lond. A 365, 665-681.

Barenblatt, G. I., Chorin, A. J. \& Prostokishin, V. M. 2000 Self-similar intermediate structures in turbulent boundary layers at large Reynolds numbers. J. Fluid Mech. 410, 263-283.

BRADSHAW, P. 1967 Inactive motions and pressure fluctuations in turbulent boundary layers. J. Fluid Mech. 30, 241-258.

CARLier, J. 2001 Etude des structures cohérentes de la turbulence de paroi à grand nombre de Reynolds par vélocimétrie par images de particules. $\mathrm{PhD}$ thesis, University of Lille.

Comte-Bellot, G. 1965 Ecoulement turbulent entre deux parois parallèles. Tech. Rep. 419, Publications Scientifiques et Techniques du Ministère de l'Air, Paris. AGARD: PCH13.

DeGraAfF, D. B. \& EATON, J. K. 2000 Reynolds number scaling of the flat-plate turbulent boundary layer. J. Fluid Mech. 422, 319-346.

Durst, F., Jovanovic, J. \& Sender, J. 1995 LDA measurements in the near-wall region of a turbulent pipe flow. J. Fluid Mech. 295, 305-335. AGARD: PCH01.

Fernholz, H. H. \& Finley, P. J. 1996 The incompressible zero-pressure-gradient turbulent boundary layer: an assessment of the data. Prog. Aerospace Sci. 32, 245-311.

Fernholz, H. H., Krause, E., Nockermann, M. \& Schober, M. 1995 Comparative measurements in the canonical boundary layer at $R e_{\delta_{2}} \leqslant 6 \times 10^{4}$ on the wall of the German-Dutch wind tunnel. Phys. Fluids 7, 1275-1281.

FLORES, O. \& JimÉNEZ, J. 2006 Effect of wall-boundary disturbances on turbulent channel flows. $J$. Fluid Mech. 566, 357-376.

Flores, O., Jiménez, J. \& del Álamo, J. C. 2007 Vorticity organization in the outer layer of turbulent channels with disturbed walls. J. Fluid Mech. 591, 145-154.

Guala, M., Hommema, S. E. \& Adrian, R. 2006 Large-scale and very-large-scale motions in turbulent pipe flow. J. Fluid Mech. 554, 521-542.

Hites, M. M. 1997 Scaling of high-Reynolds number turbulent boundary layers in the national diagnostic facility. PhD thesis, Illinois Institute of Technology.

HoXey, R. \& RichaRdS, P. 1992 Spectral characteristics of the atmospheric boundary layer near the ground. Tech. Rep., Silsoe Research Institute, UK. 
Hoyas, S. \& JimÉnEZ, J. 2006 Scaling of the velocity fluctuations in turbulent channels up to $R e_{\tau}=2003$. Phys. Fluids 18, 011702.

Hoyas, S. \& JimÉnez, J. 2008 Reynolds number effects on the Reynolds-stress budgets in turbulent channels. Phys. Fluids (to appear).

Hu, Z., Morley, C. \& Sandham, N. 2006 Wall pressure and shear stress spectra from direct simulations of channel flow. AIAA J. 44, 1541-1549.

Hutchins, N. \& Marusic, I. 2007 Evidence of very long meandering features in the logarithmic region of turbulent boundary layers. J. Fluid Mech. 579, 467-477.

Iwamoto, K., Tsukahara, T., Nakano, H. \& Kawamura, H. 2006 Effect of large-scale structures upon near-wall turbulence. In IUTAM Symposium on Computational Physics and New Perspectives in Turbulence (ed. Y. Kaneda), pp. 53-58. Springer.

JimÉnEZ, J. 2004 Turbulent flows over rough walls. Ann. Rev. Fluid Mech. 36, 173-196.

JimÉneZ, J., Del Álamo, J. C. \& FloRes, O. 2004 The large-scale dynamics of near-wall turbulence. J. Fluid Mech. 505, 179-199.

JimÉnez, J. \& Moser, R. D. 2007 What are we learning from simulating wall turbulence? Phil. Trans. R. Soc. Lond. A 365, 715-732.

KIM, J. 1989 On the structure of pressure fluctuations in simulated turbulent channel flow. J. Fluid Mech. 239, 157-194.

Kim, J., Morn, P. \& Moser, R. D. 1987 Turbulence statistics in fully developed channel flow at low Reynolds number. J. Fluid Mech. 177, 133-166.

Kolmogorov, A. N. 1941 The local structure of turbulence in incompressible viscous fluids for very large Reynolds numbers. Dokl. Akad. Nauk. SSSR 30, 301-305. Reprinted in Proc. R. Soc. Lond. A 434, 9-13 (1991).

KunKel, G. J. 2003 An experimental study of the high Reynolds number boundary layer. PhD thesis, University of Minnesota.

Kunkel, G. J. \& MARUSIC, I. 2006 Study of the near-wall-turbulent region of the high-Reynoldsnumber boundary layer using atmospheric data. J. Fluid Mech. 548, 375-402.

Lauchle, G. \& Daniels, M. 1987 Wall-pressure fluctuations in turbulent pipe flow. Phys. Fluids 30, 3019-3024.

Lawn, C. J. 1971 The determination of the rate of dissipation in turbulent pipe flow. J. Fluid Mech. 48, 477-505.

Morrison, J. F., McKeon, B. J., Jiang, W. \& Smits, A. J. 2004 Scaling of the streamwise velocity component in turbulent pipe flow. J. Fluid Mech. 508, 99-131.

Nakagawa, H. \& NezU, I. 1977 Prediction of the contributions to the Reynolds stress from bursting events in open channel flow. J. Fluid Mech. 80, 99-128.

Österlund, J. M., Johansson, A. V., Nagib, H. M. \& Hites, M. 2000 A note on the overlap region in turbulent boundary layers. Phys. Fluids 12, 1-4.

Perry, A. E., Henbest, S. M. \& Chong, M. S. 1986 A theoretical and experimental study of wall turbulence. J. Fluid Mech. 165, 163-199. AGARD: PCH02.

Perry, A. E. \& LI, J. D. 1990 Experimental support for the attached-eddy hypothesis in zeropressure-gradient turbulent boundary layers. J. Fluid Mech. 218, 405-438.

Schlichting, H. 1968 Boundary Layer Theory, sixth edn. McGraw-Hill, pp. 553-557.

SMITH, R. W. 1994 Effect of Reynolds number on the structure of turbulent boundary layers. PhD thesis, Princeton University. AGARD: TBL00.

Spalart, P. R. 1988 Direct simulation of a turbulent boundary layer up to $R e_{\theta}=1410$. J. Fluid Mech. 187, 61-98.

DER ToOnder, J. M. 1995 Drag reduction by polymer additives in a turbulent pipe flow: laboratory and numerical results. PhD thesis, Delft University of Technology. AGARD: PCH03.

Townsend, A. 1976 The Structure of Turbulent Shear Flow, 2nd edn. Cambridge Univrsity Press.

Wosnik, M., Castillo, L. \& George, W. K. 2000 A theory for turbulent pipe and channel flows. $J$. Fluid Mech. 421, 115-145.

Zagarola, M. V., Perry, A. E. \& Smits, A. J. 1997 Log laws or power laws: The scaling of the overlap region. Phys. Fluids 9, 2094-2100. 